\title{
Los Músculos Extensores Radiales del Carpo y su Importancia Clínica. Una Revisión de la Literatura
}

\author{
The Radial Carpal Extensor Muscles and their Clinical Significance. A Review of the Literature
}

\author{
Mariano del Sol ${ }^{*, * *} \&$ Aladino Cerda**,*****
}

DEL SOL, M. \& CERDA, A. Los músculos extensores radiales del carpo y su importancia clínica. Una revisión de la literatura. Int. J. Morphol., 33(3):936-941, 2015.

RESUMEN: Los extensores radiales del carpo son dos músculos de la parte lateral del compartimento posterior del antebrazo, que se utilizan con frecuencia en cirugías de transferencia de tendón. Las variaciones anatómicas de estos músculos son bastante comunes y su conocimiento es clínicamente significativo, ya que se pueden utilizar para corregir deformidades de la mano. El objetivo de este estudio es conocer la anatomía de los músculos extensores radiales, su embriología, sus variaciones anatómicas y su importancia clínica.

PALABRAS CLAVE: Músculos extensores radiales del carpo; Músculo extensor radial largo del carpo; Músculo extensor radial corto del carpo; Miembro superior; Cirugías de transferencia de tendón.

\section{INTRODUCCIÓN}

En el miembro superior, los músculos extensores radiales del carpo se utilizan a menudo en las transferencias del tendón después de lesiones nerviosas o de la médula espinal (Doyle \& Botte, 2003). Las variaciones anatómicas de estos músculos son bastante comunes y su conocimiento es clínicamente significativo en algunos procedimientos quirúrgicos como la transferencia de tendón y la corrección de las deformidades de la mano (Jetti et al., 2010).

Para realizar esta revisión se consultaron artículos en inglés y español, publicados y disponibles en las bases de datos SciELO, PubMEd y ScienceDirect. La búsqueda se realizó utilizando términos como $M$. extensor carpi radialis, $M$. extensor carpi radialis longus y M. extensor carpi radialis brevis. También se utilizaron textos de anatomía.

\section{ANATOMÍA}

Los extensores radiales del carpo, son dos músculos de la parte lateral del compartimento posterior del antebrazo. El músculo extensor radial largo del carpo (ERLC), se encuentra parcialmente cubierto por el músculo braquiorradial (BR) (Fig. 1). Es aplanado y carnoso en su tercio proximal y tendinoso en el resto de su extensión (Testut \& Latarjet, 1975). Se origina en el margen lateral del húmero, distal al músculo BR (Testut \& Latarjet), específicamente en la cresta supracondílea lateral (Lippert, 2006; Moore, 2010). Autores como Kahle et al. (1995); Latarjet \& Ruíz-Liard (2007) y Schünke et al. (2010) agregaron que también se originaba en el septo intermuscular lateral del húmero. Desde aquí sus fibras se dirigen verticalmente hacia distal y terminan, un poco más abajo del tercio proximal del antebrazo, en un tendón aplanado que continua la dirección del cuerpo muscular, pasa por el segundo compartimento del retináculo de los músculos extensores y se inserta en la cara dorsal de la base del segundo metacarpiano (Drake, 2005; Rouvière \& Delmas, 2005; Latarjet \& Ruíz-Liard; Moore et al., 2010) (Fig. 2).

El ERLC a nivel del tercio distal del antebrazo es cruzado superficialmente por el músculo abductor largo del pulgar (ALP), el músculo extensor corto del pulgar (ECP) y el músculo extensor largo del pulgar (ELP). Por su cara profunda cubre a su vez al músculo extensor radial corto del carpo (ERCC) (Testut \& Latarjet) (Fig. 1).

\footnotetext{
* Facultad de Medicina, Centro de Excelencia en Estudios Morfológicos y Quirúrgicos (CEMyQ), Universidad de La Frontera, Temuco, Chile.

** Center for Biomedical Research, Universidad Autónoma de Chile, Temuco, Chile.

*** Unidad de Anatomía, Departamento de Ciencias Básicas Biomédicas, Facultad de Ciencias de la Salud, Universidad de Talca, Talca, Chile.

***** Programa de Magíster en Ciencias, mención Morfología, Facultad de Medicina, Universidad de la Frontera, Temuco, Chile.
} 


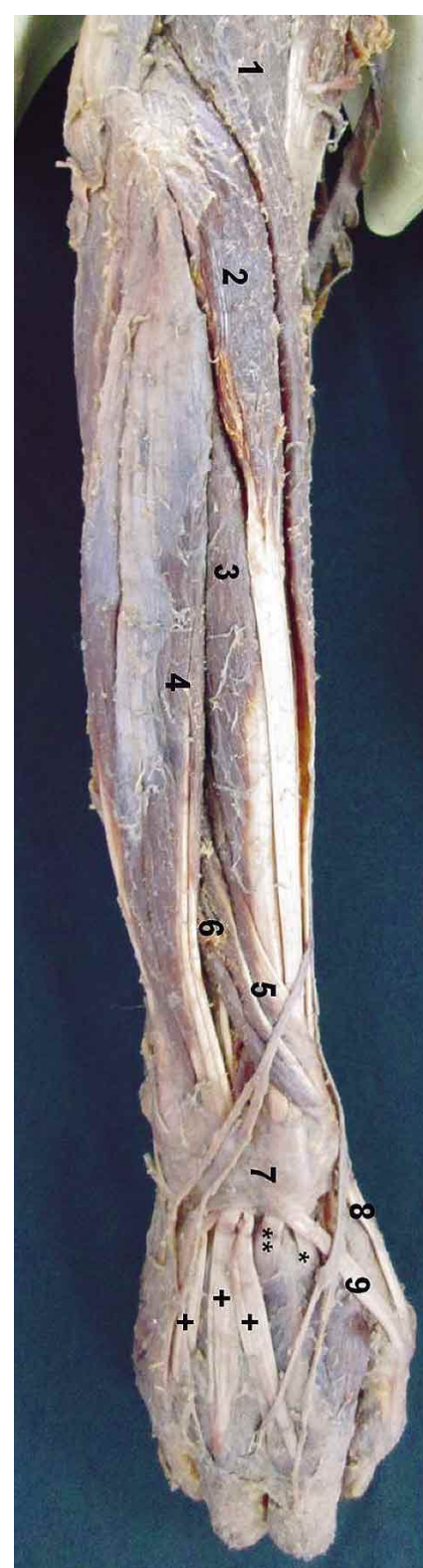

Fig. 1. Vista posterior del antebrazo y mano derecha. 1. músculo braquiorradial; 2. Músculo extensor radial largo del carpo; 3. Músculo extensor radial corto del carpo; 4. Músculo extensor de los dedos; 5. Músculo abductor largo del pulgar; 6. Músculo extensor corto del pulgar: 7. Retináculo de los músculos extensores; 8 . Tendón del músculo extensor corto del pulgar; 9 . Tendón del músculo extensor largo del pulgar; +++ Tendones del músculo extensor de los dedos; *: tendón del músculo extensor radial largo del carpo; **: tendón del músculo extensor radial corto del carpo.

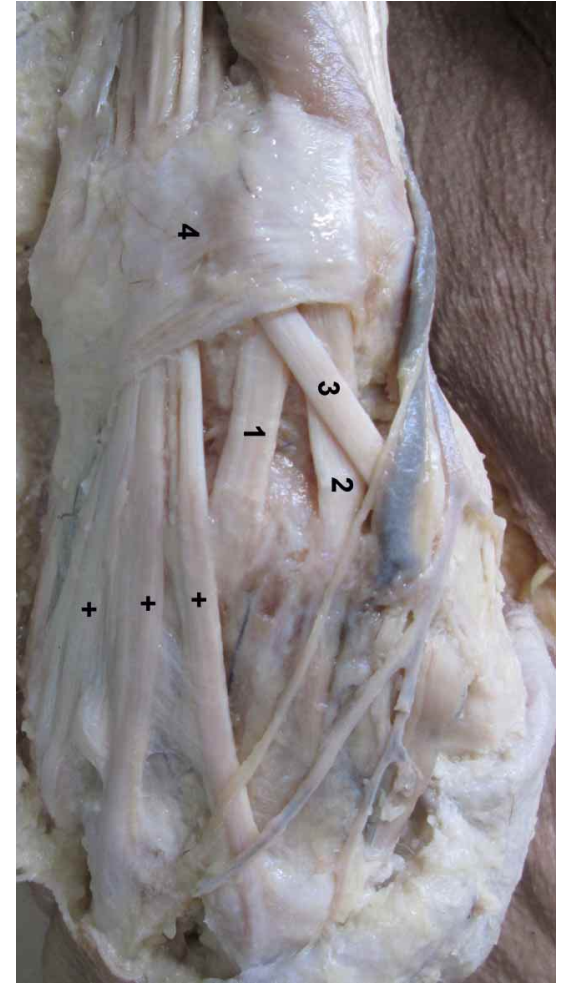

Fig. 2. Vista posterior de la mano derecha. 1. Tendón del músculo extensor radial corto del carpo; 2. Tendón del músculo extensor radial largo del carpo; 3. Tendón del músculo extensor largo del pulgar; 4. Retináculo de los músculos extensores; +++: tendones del músculo extensor de los dedos.
El ERCC, se origina en el epicóndilo lateral del húmero, junto a los músculos extensores de los dedos (Moore; Gosling, 1994). Kahle et al., agregaron que se originaba además en el ligamento anular del radio y Testut \& Latarjet, del ligamento colateral radial y el septo fibroso que lo separa del músculo extensor de los dedos (ED). Sus fibras se dirigen hacia distal y terminan, en la parte media del antebrazo, en un tendón aplanado, que acompaña al tendón del ERLC hasta la articulación radiocarpiana, pasando por el segundo compartimento del retináculo de los músculos extensores (Fig. 1). Aquí se desvía en ángulo agudo para dirigirse hacia distal y medial e insertarse en la cara dorsal de la base del tercer metacarpiano (Testut \& Latarjet) (Fig. 2).

Su cara superficial está cruzada sucesivamente por los tres músculos del pulgar: un poco más arriba de la articulación radiocarpiana, por el músculo ALP y el músculo ECP y a nivel de la articulación radiocarpiana por el músculo ELP (Testut \& Latarjet).

Ambos músculos realizan extensión y desviación radial de la articulación radiocarpiana (Schünke et al.; Pro; Moore et al.; Gosling, 1994; Drake et al.; Latarjet \& Ruíz-Liard; Testut \& Latarjet). Debido a que la inserción del músculo ERCC está próxima al eje del movimiento de desviación radial, actúa sólo de asistencia, sin embargo, es el motor primario en la extensión de la articulación radiocarpiana (Lippert, 2006). El músculo ERCC además, estabiliza la articulación radiocarpiana cuando se flexionan los dedos (al-Qattan, 1996).

Lieber et al. (1990), señalaron que la fuerza de contracción de un músculo es proporcional a su área de sección transversal fisiológica (PCSA). La gran PCSA del músculo ERCC $\left(6,94 \pm 1.84 \mathrm{~cm}^{2}\right)$ y la corta longitud de sus fibras favorecen la producción de fuerza, mientras que la PCSA más pequeña del músculo ERLC $\left(3,75 \pm 1.26 \mathrm{~cm}^{2}\right)$ y las fibras más largas, favorecen la excursión, lo que permite un mayor rango de movimiento (Ravichandiran et al., 2009). Los brazos de momento del tendón del músculo ERLC en la articulación radiocarpiana son alrededor de 1,3 cm de extensión y 1,5 cm de desviación radial, mientras que los brazos de momento en el codo van desde 1 a $5 \mathrm{~cm}$ para las diferentes fibras. Por lo tanto las fibras que se insertan más altas en el húmero tienen mucho más potencial para la flexión de la articulación cubital que para la extensión de la articulación radiocarpiana, por lo que el músculo ERLC depende de los extensores de la articulación cubital como sinergistas y es menos eficaz como extensor de la articulación radiocarpiana cuando se flexiona el codo (Brand et al., 1981). 


\section{EMBRIOLOGÍA}

Durante la evolución temprana de los tetrápodos una masa extensora común humerorradial se diferencia en los músculos BR, extensor radial común del carpo y supinador. En los anfibios y reptiles los músculos extensores radiales del carpo se insertan en los huesos radiales del carpo. En los mamíferos, la inserción migra distalmente hacia el metacarpo y el músculo se divide en dos, el músculo ERLC y el músculo ERCC, que se insertan en las caras dorsales de las bases del segundo y tercer metacarpiano, respectivamente (BojsenMoller, 1978).

La división del músculo extensor radial común del carpo en un músculo ERLC y un músculo ERCC, está presente en Primates, la Tulapidae, los Galeopithques, la mayoría de los roedores, algunos carnívoros y algunos marsupiales. Por otra parte, en los insectívoros, muchos carnívoros, algunos roedores, el Lagomorpha, el Dasypodidae, los Pholidotes y algunos marsupiales, los dos músculos, son de hecho uno (Cossu \& Guintard, 2000).

De acuerdo con Williams et al. (1989), dado que ambos músculos se originan de un blastema común, sus vientres pueden estar parcialmente fusionados o conectados por haces adicionales, lo que explica la frecuencia de sus variaciones.

\section{VARIACIONES ANATÓMICAS}

Numerosos autores han reportado la presencia de músculos accesorios que se originan con los músculos extensores radiales del carpo, tendones accesorios, variaciones en su inserción y bandas de interconexión.

Wood (1867) adoptó nombres para los músculos adicionales que encontró durante sus disecciones: Extensor radial accesorio del carpo (ERAC), para aquellos que se insertan en el pulgar y el de extensor radial intermedio del carpo (ERIC), para aquellos que se originan con el músculo ERLC o el músculo ERCC.

El término extensor radial accesorio del carpo fue dado a una variación o la división del músculo ERLC, con un curso a través del mismo compartimento del retináculo de los músculos extensores, pero con una inserción en la base del primer metacarpiano (Wood, 1867). Existe una baja incidencia de estos músculos accesorios, por lo que su presencia sólo se ha dado a conocer en reportes aislados (Hong \& Hong, 2005).

El término extensor radial intermedio del carpo fue otorgado por Wood (1867) a aquel músculo que se origina entre los músculos extensores radiales del carpo y se inserta en la base del segundo o tercer metacarpiano. Se encuentra entre el 12\% (39 de 312 miembros superiores) (Wood, 1988) y el $24 \%$ de los casos (42 de 173 miembros superiores) (Albright \& Linburg, 1978).

Wood (1866) reportó la coexistencia del ERIC y el ERAC. Chakravarthi (2013) describió un ERLC adicional que se insertaba en la base del segundo metacarpiano y Vessal \& Rai (2006) un ERCC adicional derivado del tendón distal del ERCC.

En el 36\% de los 156 cadáveres examinados por Wood (1988) se encontraron tendones adicionales (140 tendones accesorios en 111 miembros superiores). De éstos, 29 fueron desde el músculo ERLC y se insertaron en el tercer metacarpiano con el tendón del ERCC; 24 se originaron del ERLC y se insertaron junto al tendón principal en el segundo metacarpiano y 7 se originaron en el vientre del ERCC y se insertaron en el tercer metacarpiano. En el 26\% de los 173 miembros superiores disecados por Albright \& Linburg se encontró un tendón adicional del ERLC que se insertaba distalmente con el ERCC, y en el 21\%, un tendón del ERCC que se insertaba con el ERLC. Caetano et al. (2004) encontraron dos tendones del músculo ERCL, en tres de las 60 disecciones. Prakash et al. (2008) reportaron que en uno de los 200 miembros superiores disecados, el ERCC no estaba presente, mientras que el ERLC presentó dos tendones. Claassen \& Wree (2002) describieron en un cadáver femenino la presencia de una cabeza accesoria del músculo ERLC y un tendón accesorio del músculo ERCC.

Entre las disecciones realizadas por Wood (1867), en dos sujetos el tendón del ERCC se insertaba en el margen ulnar de la base del segundo y tercer metacarpiano.

En cuatro muestras del estudio realizado por Caetano et al., se encontró la presencia de una banda de interconexión uniendo los tendones de los músculos extensores radiales del carpo. Este tendón intermedio (llamado así por Testut \& Latarjet), se extendía desde el tendón del músculo ERLC al tendón del músculo ERCC. Wood (1865) describió en un sujeto los músculos extensores radiales del miembro superior izquierdo intercambiando fascículos tendinosos. Gümüsalan et al. (1997) observaron en un cadáver femenino de 55 años la presencia de una banda muscular en ambos antebrazos.

\section{IMPORTANCIA CLÍNICA DE LOS MÚSCULOS EXTENSORES RADIALES DEL CARPO Y SUS VA- RIACIONES}

Los músculos extensores radiales del carpo son extensamente usados en cirugías de transferencia de tendón, especialmente para la restauración de los movimientos del 
pulgar (Georgiev et al., 2006.). Dicha transferencia es un procedimiento quirúrgico que implica mover la inserción de una unidad músculo-tendón de un lugar a otro (HerasPalou \& Burke, 2003).

Cooney et al. (1984) estudiaron varios músculos para lograr la transferencia efectiva de tendón en la parálisis del nervio mediano. Encontraron que el ERLC es uno de los mejores en la aproximación de la fuerza y el movimiento necesario para restaurar la pérdida de la flexión del pulgar, la oposición completa del pulgar y la fuerza tanto en la parálisis alta y baja del nervio mediano.

Es conocido que los músculos tenares que participan en la oposición del pulgar son el ACP, el oponente del pulgar (OP) y la cabeza superficial del músculo flexor corto del pulgar (FCP), y que la fracción de la tensión de estos músculos es de 3,8\% (PCSA $6.0 \mathrm{~cm}^{2}$ ) (Baek et al., 1999). De acuerdo con Cooney et al., la fracción de la tensión del ERLC es de $3,1 \%$. Brand et al. indicaron que la fracción de tensión del ERCC es de 4,2\%; ambos por lo tanto, tienen la tensión muscular suficiente para sustituir la fuerza de la oposición perdida.

Según Cooney et al., el tendón para el dedo anular del músculo flexor superficial de los dedos y de los músculos extensores de la articulación radiocarpiana (Extensor ulnar del carpo (EUC), ERLC) son los que mejor aproximan la fuerza y el movimiento necesario para la oposición completa del pulgar. El ERLC y el tendón para el dedo anular del FSD tendrían aproximadamente $80 \%$ y $60 \%$, respectivamente, de la fuerza muscular tenar requerida.

Numerosas técnicas de transferencia de tendón se han ideado para restaurar la aducción del pulgar y corregir la deformidad de la mano en garra en la parálisis del nervio ulnar (Park et al.). Brand et al., indicaron que en términos de fuerza muscular, el FSD, EUC, ERLC y ERCC son adecuados para restaurar la aducción del pulgar. Si se transfiere un músculo extensor de articulación radiocarpiana al aductor del pulgar, se libera al músculo flexor superficial de los dedos, que puede a continuación, ser utilizado para corregir otras deformidades de la parálisis del nervio ulnar tal como los dedos en garra (Fischer et al., 2003). Baek et al., indicaron que como el EUC extiende y desvía ulnarmente la articulación radiocarpiana, si es que se utiliza para transferencia de tendón, la articulación radiocarpiana se extendería sólo en desviación radial. Sin embargo, cuando se utiliza uno de los dos extensores radiales, la articulación radiocarpiana se puede extender en posición neutra, desviación radial y ulnar.

Los extensores radiales del carpo han sido también utilizados para la restauración de la flexión de los dedos en pacientes con pérdida de los músculos flexores después de un trauma directo (Sabapathy et al., 2005); para la transferencia de tendón en la mano reumatoide (Boyce et al., 1978); en la restauración de la pinza (Fischer et al.) y mejorar la función de la mano después de la parálisis irreparable del nervio ulnar (Ozcan et al., 2003).

Respecto a las variaciones anatómicas de los músculos extensores radiales del carpo, estos músculos accesorios y sus tendones pueden ser utilizados para la transferencia de tendón. Albright \& Linburg señalaron que cuando la detección de un músculo adicional produciría una diferencia crítica en la función, como en pacientes con parálisis severas, los extensores radiales de la articulación radiocarpiana deben ser explorados desde su origen hasta su inserción.

Wood (1988) indicó que es necesario buscar músculos accesorios o bandas tendinosas accesorias, sobre todo en pacientes con cuadriplejía, ya que se pueden utilizar para restaurar la oposición, como motor del músculo flexor largo del pulgar, o como motor para el músculo extensor largo del pulgar. De acuerdo a su estudio, en el $12 \%$ de los personas el ERIC tendría un buen tendón y aproximadamente el 36\% de las personas tienen al menos una, y algunas veces varios tendones accesorios que serían adecuados para la transferencia. Según Vessal \& Rai, la conciencia de la existencia de los músculos accesorios es esencial para los radiólogos en ayudar a formular el diagnóstico diferencial de una masa en el miembro superior, ya que estos músculos accesorios pueden ser confundidos con un tumor.

\section{CONCLUSIÓN}

Los músculos extensores radiales del carpo son ampliamente usados en cirugías de transferencia de tendón por su fácil abordaje y porque si se utiliza sólo uno de ellos (ECRL o ERCC), no dejan secuelas significativas en el sitio donante.

El conocimiento de las variaciones anatómicas de estos músculos es importante clínicamente, por su alta prevalencia y porque pueden aumentar significativamente la funcionalidad de los pacientes que requieren múltiples transferencias de tendón. Esta revisión, entrega información detallada acerca de la anatomía de los músculos extensores radiales del carpo, su embriología, sus variaciones y su importancia clínica, la cual puede ser utilizada por profesionales de la salud que maniobran a este nivel, ya que a la palpación, los músculos accesorios podrían ser confundidos con una masa tumoral. 
DEL SOL, M. \& CERDA, A. The radial carpal extensor muscles and their clinical significance. A review of the literature. Int. J. Morphol., 33(3):936-941, 2015.

SUMMARY: Extensor carpi radialis are two lateral muscles of the posterior compartment of the forearm which are often used in tendon-transfer surgery. The anatomical variations of these muscles are quite common and knowledge about these variations is clinically significant, as it is useful to correct deformities of the hand. The aim of this study is to know the anatomy of the extensor radial muscles, embryology, anatomical variations and their clinical significance.

KEY WORDS: Musculus extensor carpi radialis; Musculus extensor carpi radialis longus; Musculus extensor carpi radialis brevis; Upper limb; Tendon transfer surgery.

\section{REFERENCIAS BIBLIOGRÁFICAS}

al-Qattan, M. M. The nerve supply to extensor carpi radialis brevis. J. Anat., 188(Pt. 1):249-50, 1996.

Albright, J. A \& Linburg, R. M. Common variations of the radial writs extensors. J. Hand Surg. Am., 3(2):134-8, 1978.

Baek, G. H.; Jung, J. M.; Yoo, W. J. \& Chung, M. S. Transfer of extensor carpi radialis longus or brevis for opponensplasty. J. Hand Surg. Br., 24(1):50-3, 1999.

Bojsen-Moller, F. Extensor carpi radialis longus muscle and the evolution of the first intermetacarpal ligament. Am. J. Phys. Anthropol., 48(2):177-84, 1978.

Boyce, T.; Youm, Y.; Sprague, B. L. \& Flatt, A. E. Clinical and experimental studies on the effect of extensor carpi radialis longus transfer in the rheumatoid hand. J. Hand Surg. Am., 3(4):390-4, 1978.

Brand, P. W.; Beach, R. B.; \& Thompson, D. E. Relative tension and potential excursion of muscles in the forearm and hand. J. Hand Surg. Am., 6(3):209-19, 1981.

Caetano, M. F.; Albertoni, W. M.; Caetano, E. B. \& Pérez, R. M. Anatomical study of insertions of the extensor carpi radialis longus and brevis. Int. J. Morphol., 22(4):245-51, 2004.

Chakravarthi, K. An additional extensor carpi radialis longus muscle and its clinical significance. Int. J. Bioassays, 2(6):887-8, 2013.

Claassen, H. \& Wree, A. Multiple variations in the region of Mm. extensores carpi radialis longus and brevis. Ann. Anat., 184(5):489-91, 2002.

Cooney, W. P.; Linscheid, R. L. \& An, K. N. Opposition of the thumb: an anatomic and biomechanical study of tendon transfers. J. Hand Surg. Am., 9(6):777-86, 1984.

Cossu, F. \& Guintard, C. Anatomical and descriptive study of the radial extensor muscle (M. extensor carpi radialis). Anat. Histol. Embryol., 29(6):337-43, 2000.
Doyle, J. R. \& Botte, M. J. Surgical Anatomy of the Hand and Upper Extremity. Philadelphia, Lippincott Williams \& Wilkins, 2003.

Drake, R. L.; Vogl, W. \& Mitchell, A. W. M. Gray. Anatomía para Estudiantes. 3a ed. Barcelona, Elsevier, 2005.

Fischer, T.; Nagy, L. \& Buechler, U. Restoration of pinch grip in ulnar nerve paralysis: extensor carpi radialis longus to adductor pollicis and abductor pollicis longus to first dorsal interosseus tendon transfers. J. Hand Surg. Br., 28(1):28-32, 2003.

Georgiev, G.; Surchev, L. \& Jelev, L. Transposition of parts between teh extensor carpi radialis muscles and their significance for han surgery. Bul. J. Orthop. Trauma., 43(3):130-4, 2006.

Gosling, J. A. Anatomía Humana. 2a ed. Madrid, Mosby/Doyma Libros, 1994.

Gümüsalan, Y.; Kalaycioglu, A.; Yazar, F.; Arifoglu, Y. \& Sinav, A. Accessory extensor carpi radialis muscle and interconnecting muscular bundle. Acta Anat. (Basel), 159(1):57-60, 1997.

Heras-Palou, C. \& Burke, F. D. Principles of tendon transfer in the hand and forearm. Curr. Orthop., 17(1):8-16, 2003.

Hong, M. K. \& Hong, M. K. An uncommon form of the rare extensor carpi radialis accessorius. Ann. Anat., 187(1):8992, 2005.

Jetti, R.; Nair, V.; Nair, R. V.; Mookambica, R. V. \& Somayaji, $\mathrm{K}$. Variant insertion of extensor carpi radialis longus in a South Indian cadaver. Int. J. Anat. Var., 3:86-7, 2010.

Kahle, W.; Leonhardt, H. \& Platzer, W. Atlas de anatomía para estudiantes y médicos: Aparato locomotor. $3^{\mathrm{a}} \mathrm{ed}$. Barcelona, Omega, 1995.

Latarjet, M. \& Ruíz-Liard, A. Anatomía Humana. $4^{\text {a }}$ ed. Buenos Aires, Médica Panamericana, 2007. 
Lieber, R. L.; Fazeli, B. M. \& Botte, M. J. Architecture of selected wrist flexor and extensor muscles. J. Hand Surg. Am., 15(2):244-50, 1990.

Lippert, L. S. Clinical Kinesiology and Anatomy. 4th ed. Philadelphia, F. A. Davis Company, 2006.

Moore, K. L.; Dalley, A. F. \& Agur, A. M. R. Anatomía con Orientación Clínica. 6a ed. Baltimore: Lippincott Williams \& Wilkins, 2010.

Ozkan, T.; Ozer, K. \& Gülgönen, A. Three tendon transfer methods in reconstruction of ulnar nerve palsy. J. Hand Surg. Am., 28(1):35-43, 2003

Park, J. S.; Baek, G. H. \& Gong, H. S. Modified extensor carpi radialis brevis adductorplasty for ulnar nerve palsy. Tech. Hand Up Extrem. Surg., 16(2):86-90, 2012.

Prakash; Rai, R.; Ranade, A. V.; Prabhu, L. V.; Pai, M. M. \& Singh, G. Multiple variations of extensor muscles of forearm in relation to the radial nerve: A case report and review. Int. J. Morphol., 26(2):447-9, 2008.

Ravichandiran, K.; Ravichandiran, M.; Oliver, M. L.; Singh, K. S.; McKee, N. H. \& Agur, A. M. Determining physiological cross-sectional area of extensor carpi radialis longus and brevis as a whole and by regions using $3 \mathrm{D}$ computer muscle models created from digitized fiber bundle data. Comput. Methods Programs Biomed., 95(3):203-12, 2009.

Rouvière, H. \& Delmas, A. Anatomía Humana: Descriptiva, Topográfica y Funcional. 11a ed. Barcelona, Masson, 2005.

Sabapathy, S. R.; Gowda, D. K.; Ranade, A. B.; Venkatramani, H. \& Sebastin, S. J. Functional outcome of extensor carpi radialis longus transfer for finger flexion in posttraumatic flexor muscle loss. J. Hand Surg. Am., 30(2):267-72, 2005.

Schünke, M.; Schulte, E.; Schumacher, U.; Voll, M. \& Wesker, K. Prometheus. Texto y Atlas de Anatomía. $2^{\mathrm{a}}$ ed. Buenos Aires, Médica Panamericana, 2010.

Testut, L. \& Latarjet, A. Compendio de Anatomía Descriptiva. 27a ed. Barcelona, Salvat Editores, 1975.

Vessal, S. \& Rai, S. B. Accessory extensor carpi radialis brevis muscle, a pseudomass of the distal forearm: ultrasound and MR appearances -case report and literature review. Clin. Radiol., 61(5):442-5, 2006.

Williams, P. L.; Warwick, R.; Dyson, M. \& Bannister, L. H. Gray's Anatomy. 37th ed. Edinburgh, Churchill Livingstone, 1989.

Wood, J. Additional varieties in human myology. Proc. R. Soc. Lond., 14:379-92, 1865.
Wood, J. Variations in human myology observed during the winter session of 1865-66 at King's College, London. Proc. R. Soc. Lond., 15:229-44, 1866.

Wood, J. Variations in human myology observed during the winter session of 1866-67 at King's College, London. Proc. R. Soc., 15:518-46, 1867.

Wood, V. E. The extensor carpi radialis intermedius tendon. $J$. Hand Surg. Am., 13(2):242-5, 1988.

Dirección para Correspondencia:

Prof. Dr. Mariano del Sol

Facultad de Medicina

Universidad de La Frontera

Temuco

CHILE

Email: mariano.delsol@ufrontera.cl

Recibido : 18-03-2015

Aceptado: 25-05-2015 